\title{
Primary hydatid cyst in an unexpected localization
}

\author{
Ergul E, Ucar AE,Yalcin S \\ Ankara Ataturk Teaching and Research Hospital, General Surgery Department, Ankara, Turkey. \\ dreergul@gmail.com
}

\begin{abstract}
Echinococcal disease remains a problem within some endemic areas. It usually involves the liver and lungs, but any other organ can potentially be involved. Soft tissue hydatid disease without liver and lung involvement was reported in $2.3 \%$ of patients. This paper presents a case of 35-year-old male patient who was referred to our department with a $9 \times 9 \times 6 \mathrm{~cm}$ hydatid cyst at psoas muscle. The rarity of the musculoskeletal disease renders the decision making on the favorable treatment quite difficult. Conservative treatment, complete excision and simple drainage have all been suggested as adequate. We advocate total en bloc excision whenever possible (Fig. 2, Ref. 4). Full Text in free PDF www.bmj.sk.

Key words: hydatid cyst, musculoskeletal, Primer, Psoas muscle.
\end{abstract}

Human hydatid disease is caused by the larval form of the tapeworm genus Echinococcus. Hydatid disease is endemic in the Mediterranean Countries, the Middle and Far East and South America. Humans are infected either by a direct contact with a dog or by ingestion of foods contaminated by the dog feces ${ }^{1}$. While hydatid cysts may occur in any area of the body, isolated muscular localizations are rare. Only some thirty-odd of such cases have been reported.

\section{Case Report}

A 35-year-old male patient was referred to the general surgery department with 6 month history of left flank pain and a mass in psoas muscle which was detected at computerized tomographic examination (Fig. 1). There was no palpable mass on physical examination. Liver tests and other laboratory tests revealed no abnormalities. Echinococcus immune hemaglutination test was positive. A 9x9x6cm cyst was removed by extraperitoneal transverse laparotomy (Fig. 2). Histopathological examination was also reported as cyst hydatid. There were no signs of hepatic, pulmonary or any other site hydatid disease. Thus, it was considered as primary psoas cyst hydatid.

\section{Discussion}

Echinococciasis or hydatid disease is a cosmopolite antropozoonosis common to humans and several mammal species. The disease results from the development of the larval or hydatid form of the canine tenia (Echinococcus granulosis) in the organism.

Ankara Ataturk Teaching and Research Hospital, General Surgery Department, Ankara, Turkey

Address for correspondence: E. Ergul, Dr, Askaabat Cad. Eser Sitesi B3 Blok, Daire:11 06490 Bahcelievler, Ankara, Turkey.

Phone: +905.056821500, Fax: +903.122123414

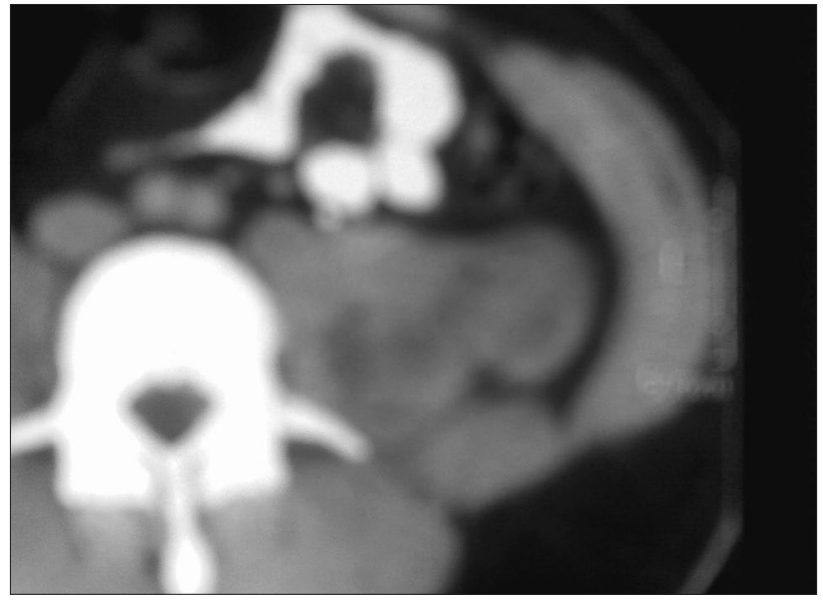

Fig. 1. A cystic mass in the left psoas muscle.

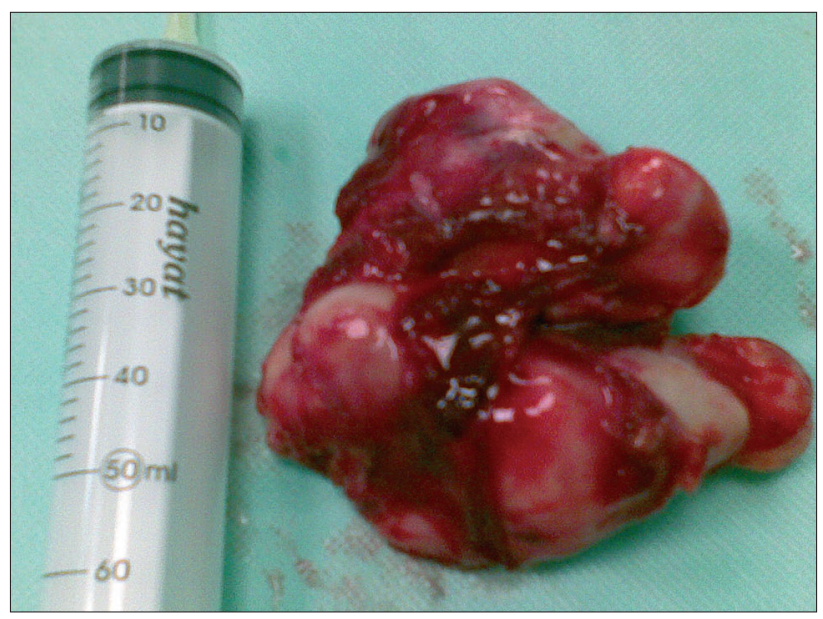

Fig. 2. Mass excisedfrom the left psoas muscle. 
Hydatid cysts are uncommonly found in muscles, even in endemic zones. After ingestion, the eggs are freed from their coating and larvae penetrate the mucosa of the jejunum, reaching through the venous and lymphatic channels every region of the body. Most larvae are trapped into the liver, which functions as a barrier. In our case, the larvae passed the liver and migrated to the psoas muscle.

Several factors may explain the exceptional nature of muscle localizations of hydatid cysts: efficacy of the hepatic and pulmonary barriers, muscle environment not favorable for growth of hydatid larvae. The predominant localization in the proximal muscles of the lower limbs could be explained by the volume of the muscle mass and its rich blood supply.

The cysts may be single or multiple, uni- or multiloculated and thin or thick walled. Soft tissue hydatid disease without liver and lung involvement occurs in $2.3 \%$ of patients. A palpable mass is the most constant clinical or exploratory finding of hydatid disease affecting soft tissues. The clinical manifestations are caused by compression of the involved organ. The growth of the hydatid cysts is usually slow and the annual growth rate of the cyst is about 1 to $3 \mathrm{~cm}$ in diameter (2). The clinical evolution of hydatid disease is non-specific. It depends on the number, dimensions and localization of the cysts. Abscess, chronic hematoma, synovial cyst and necrotic malignant soft tissue tumor should be taken into consideration in the differential diagnosis.

Ultrasonography is the diagnostic tool of choice. Our patient was referred to us with a CT scan. Thus, ultrasonographic exami- nation was not performed. It is important to establish the diagnosis preoperatively in order to limit the risk of anaphylactic shock or dissemination in the event of puncture or accidental opening of the cyst during resection.

Surgery is required for treatment, ideally by en bloc total pericystectomy. Medical treatment with imidazoles has little efficacy for the treatment of muscular hydatid disease (3). Moreover, the rarity of the musculoskeletal disease renders the decision making on the favorable treatment quite difficult. Conservative treatment, complete excision and simple drainage have all been suggested as adequate (4). We have good results with en bloc total pericystectomy. Thus, we advocate total excision whenever possible.

\section{References}

1. Kiyak G, Ergul E, Korukluoglu B. Primary hydatid disease of the soft tissue. Acta Chir Belg, 2007; 107: 452-453.

2. Sayek I, Tirnaksiz B, Dogan R. Cystic hydatid disease: Current trends in diagnosis and management. Surg Today 2004; 34: 987-996.

3. Mseddi M, Mtaoumi M, Dahmene J, Ben Hamida R, Siala A, Moula T, Ben Ayeche ML. Hydatid cysts in muscles: eleven cases. Rev Chir Orthop Reparatrice Appar Mot 2005; 91 (3): 267-271.

4. Drimousis PG, Stamou KM, Koutras A, Tsekouras DK, Zografos G. Unusual site of recurrent musculoskeletal hydatid cyst: Case report and Brief review of the literature. World J Gastroenterol 2006; 12 (34): 5577-5578. 